\title{
Erratum to: Viscous flows with flat free boundaries
}

\section{V. Pukhnachev ${ }^{1,2, a}{ }_{\mathbb{D}}$, E. N. Zhuravleva ${ }^{1,2}{ }_{\mathbb{D}}$}

${ }^{1}$ Lavrentyev Institute of Hydrodynamics of the Siberian Branch of the Russian Academy of Sciences, Novosibirsk, Russia

2 Novosibirsk State University, Novosibirsk, Russia

(C) The Author(s), under exclusive licence to Società Italiana di Fisica and Springer-Verlag GmbH Germany, part of Springer Nature 2021

\section{Erratum to: Eur. Phys. J. Plus (2020) 135:554 https://doi.org/10.1140/epjp/s13360-020-00552-z}

The original version of this article contains a mistake.

The correct form of the equation (11) is:

$$
\frac{d^{3} \psi}{d \xi^{3}}+\left(\frac{\xi}{2}-\psi\right) \frac{d^{2} \psi}{d \xi^{2}}+\left(\frac{d \psi}{d \xi}\right)^{2}+\frac{d \psi}{d \xi}=0 .
$$

The graph of function $\psi(\xi)$ shown in Figure 2 is based on the correct equation.

The original article can be found online at https://doi.org/10.1140/epjp/s13360-020-00552-z.

a e-mail: pukhnachev@gmail.com (corresponding author) 